\title{
Genome-Wide Bisulphite Sequencing Uncovered the Contribution of DNA Methylation to Rice Short-Term Drought Memory Formation
}

\author{
Shuyan Kou ${ }^{1}$ Q Qiongyao Gu ${ }^{1}$ Liu Duan ${ }^{2}$ - Gaojing Liu ${ }^{3}$ Pingrong Yuan ${ }^{1} \cdot$ Huahui Li ${ }^{1} \cdot$ Zhigang Wu$^{1} \cdot$ Weihua Liu ${ }^{1}$. \\ Ping Huang ${ }^{1} \cdot$ Li Liu $^{2}$ (D)
}

Received: 17 May 2021 / Accepted: 26 August 2021 / Published online: 7 September 2021

(C) The Author(s) 2021

\begin{abstract}
Rice is the important crop for more than half of the world population. However, drought can have a devastating impact on rice growth and reduce yield drastically. Understanding the response of rice to drought stress is especially important for crop breeding. Previously we found that rice enhanced its tolerance to drought stresses via stress memory mechanisms. Numerous memory genes were identified to play important roles in the process. DNA methylation was reported to mediate tolerance via regulating gene expression and enhances the survival rate of rice encountering drought stress. However, how DNA methylation involved in stress memory is still not clear. In this study, genome-wide bisulphite sequencing at a single base resolution methylome profiling level was performed and analyzed in rice cultivar under recurrent drought stresses and recovery treatments. We found that rice drought stress memory-related differentially methylated regions (DMRs) showed dynamic and distinct patterns. The drought-memory DMRs may regulate Transposable elements and gene expression to cope with short-term repeated drought stresses. Our findings of drought-memory DMRs can explain mechanisms of rice drought stress memory in a new perspective on global methylome details. Using epigenetic markers to breed drought-resistant rice would become a feasible way in the future research.
\end{abstract}

Keywords Oryza sativa $\cdot$ Drought stress $\cdot$ DNA methylation $\cdot$ Short-term epigenetic memory $\cdot$ Stress memory

Handling editor: Mikihisa Umehara.

Shuyan Kou, Qiongyao Gu, Liu Duan and Gaojing Liu have contributed equally to this work.

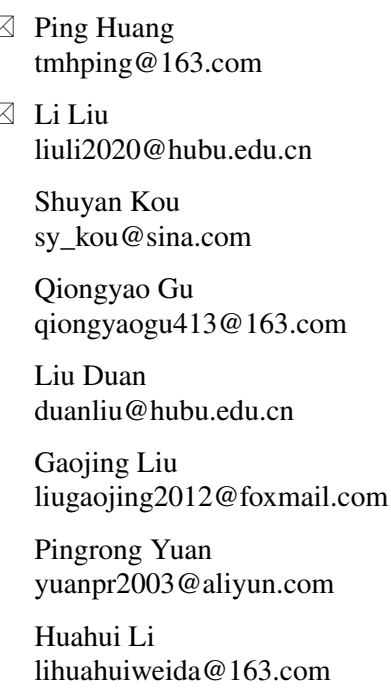

\author{
Zhigang Wu \\ wuzhigangswu@163.com \\ Weihua Liu \\ lwhynu@126.com \\ 1 Institute of Food Crop, Yunnan Academy \\ of Agriculture Science, Kunming 650205, Yunnan, \\ People's Republic of China \\ 2 State Key Laboratory of Biocatalysis and Enzyme \\ Engineering, Hubei Collaborative Innovation Center \\ for Green Transformation of Bio-Resources, Hubei \\ Key Laboratory of Industrial Biotechnology, School \\ of Life Sciences, Hubei University, Wuhan, Hubei, \\ People's Republic of China \\ 3 Berry Oncology Corporation, Fuzhou 350200, \\ People's Republic of China
}




\section{Introduction}

Rice is a major staple food crop that contributes to the diets of half of the world's inhabitants, especially in Asia. However, multiple environmental stresses, including drought, flooding, high temperature, salinity, among others, affect crop productivity and sustainability of agriculture. Drought massively affects the growth and yield of rice, which is a semiaquatic plant and considered as one of the most drought-susceptible plants. Almost all the developmental stages of rice can be affected by drought, especially the reproductive stage, resulting in severe yield penalties. Therefore, it is urgently needed to breed drought-resistant/tolerant rice for improving rice yield under drought to meet with the increasing population and the drought challenge that occurs. Understanding the mechanisms of how rice responds and adapts to drought stress will provide us grounds for optimism.

Rice employs complicated processes and multiple strategies in response to drought, involves various physiological, biochemical, and genetic responses. Many traits under drought have been reported, such as modification of cell wall plasticity, root extension, leaf area, and surface properties (Ganie and Ahammed 2021; Kim et al. 2020a, b), etc. Multiple ways at genetic levels, transcriptomic levels, and proteomic levels were extensively used to reveal the drought stress genes and pathways. Currently, several transcriptional factors (TFs) are known to play crucial roles in regulation of a series of downstream genes (e.g., cell expansion and cell wall-related genes, lignin biosynthetic gens, cellulose synthase) to cope with drought, such as AP2/ERF family TF genes (Jung et al. 2017; Lee et al. 2016), the NAC group of TFs(NAM, ATAF, and CUC) (Hu et al. 2006; Nakashima et al. 2007; Redillas et al. 2012; Zheng et al. 2009), WRKYs (Sahebi et al. 2018; Shen et al. 2012). MicroRNAs (miRNAs), which regulate diverse developmental and stress-related processes, are regarded to modulate these traits as well (Zhang et al. 2018b; Nadarajah and Kumar 2019). Various components of ABA signaling, plant hormones crosstalk (Todaka et al. 2015; Riemann et al. 2015; Santosh Kumar et al. 2020), and metabolic networks (Fàbregas and Fernie 2019) are well described in response to drought stress in different species. Other than these, DNA methylation, which is one of the main types of epigenetic modifications that commonly presented in eukaryotic genomes and significantly regulate gene expression, also play a critical role in response to plant drought stresses (Li et al. 2020).

DNA methylation occurs at sequence contexts of $\mathrm{CG}$, $\mathrm{CHG}$, and $\mathrm{CHH}(\mathrm{H}=\mathrm{A}, \mathrm{C}$, or $\mathrm{T})$. $\mathrm{CG}$ sites methylation are usually mediated by methyltranserase 1 (MET1), while CHG sites are mediated by chromomethylase 3 (CMT3), and $\mathrm{CHH}$ sites are governed by domains rearranged methyltransferase 1/2 (DRM1/2) and chromomethylase 2 (CMT2) (Kankel et al. 2003; Law and Jacobsen 2010; Lindroth et al. 2001). Using Methylation sensitive amplified polymorphism technique, hypermethylation was found dominant in drought-susceptible rice genotypes IR20 and CO43, while hypomethylation was found to be presented in drought-tolerant rice genotypes PL and PMK3 when under drought condition (Gayacharan 2013). 68 rice accessions of osmotic stress-tolerant and -susceptible groups were further checked for their DNA methylation levels, with similar conclusion that drought-tolerant accessions possessed lower DNA methylation and more de-methylation events under stressed condition (Xia et al. 2017). A latter study using methylated DNA immunoprecipitation sequencing and Affymetrix GeneChip array also reported that differentially methylated regions (DMRs) in droughttolerant introgression line DK151 was far more less than DMRs in drought-sensitive line IR64, which suggested drought-tolerant rice plants have a more stable methylome (Wang et al. 2016). DNA methylation was positively correlated with spikelet sterility and negatively correlated with yield, and has various effects on gene expression (Gayacharan 2013; Wang et al. 2016). A recent study also reported that methylation in $\mathrm{CG}$ and $\mathrm{CHH}$ contexts within gene body and distal promoter regions were positively correlated with the expression of abiotic stress response genes (Rajkumar et al. 2020). Hypomethylation of Transposable elements (TEs) in plants were associated with transcriptional/transpositional activities (Hu et al. 2014; Miura et al. 2001). Methylation of TEs nearby genes affect their expression levels. TEs were found methylated mostly (>80\%) in CG sites, and 20-90\% in CHG sites, $2-30 \%$ in $\mathrm{CHH}$ sites (Niederhuth et al. 2016). CG, $\mathrm{CHG}$, and $\mathrm{CHH}$ methylation in $A$. thaliana TEs averages at about $80 \%$, $40 \%$, and $15 \%$, respectively. $\mathrm{CG}, \mathrm{CHG}$, and $\mathrm{CHH}$ methylation in $P$. patens TEs averages around $80 \%, 80 \%$, and $30 \%$, respectively (Domb et al. 2020). DRMs targeted to TEs via RNA-directed DNA methylation pathway, and methylation under all three contexts are important for TE silencing. These evidence supported that DNA methylation is an important epigenetic regulatory mechanism for rice to adapt drought stress.

However, throughout its whole life, plants can constantly suffer from environmental fluctuations such as drought, which might occur in a short time (transient) or over long periods. Plants submitted to repeated stress treatments responded differently than to a single stress event, which called "stress memory". Stress memory can trigger physiological and molecular processes and help plants to enhance their resistance to stress. Many plants were found to exhibit memory behavior, such as switchgrass (Zhang et al. 2018a, b), Zea mays (Virlouvet et al. 2018), and rice (Li et al. 2019), Brazilian savanna (Alves 
et al. 2020), Glycine max L. (Kim et al. 2020a, b), Pinus pinaster (Fernández de Simón et al. 2020). Exposure to drought resulted in several changes in plants, and these changes in plants submitted to recurrent drought stress differed when compared in a single drought event. This helps in acclimation to drought stress for plant survival, involving several metabolic pathways, gene expression changes, and physiological and phytohormones changes. Proline has been considered to be a critical component of stress memory, including drought (Li et al. 2019). The free proline levels increased by the first drought stress and remained stable throughout the subsequent drought treatment, in corresponding to the gene expression of proline biosynthetic enzyme $\Delta 1$-pyrroline-5-carboxylate synthetase 1 (P5CS1) in rice ( $\mathrm{Li}$ et al. 2019). Besides, DNA methylation was also believed to participate in rice short-term drought memory, although the mechanism was not clear. Multiple ways were employed to study the plant epigenetic stress memory (Godwin Farrona 2020). By methylation-sensitive amplification polymorphism (MSAP) method, both droughtsensitive and -resistant varieties showed a cumulative effect on DNA methylation pattern from the original generation to the sixth generation, which suggested that some DNA methylation status induced by drought stress could inherit and transmit to the next generation (Zheng et al. 2013). Wheat seeds from terminal drought stressed plants showed better growth than the progenies of well-watered crop, and seed priming improved drought tolerance (Tabassum et al. 2018). Meanwhile, rice also was found exhibited short-term drought stress memory in response to cycles of mild drought and re-watering, and several genes involved in rice drought memory response were identified through a whole-transcriptome strand-specific RNA sequencing ( $\mathrm{Li}$ et al. 2019). A linkage was found between drought memory transcripts and DNA methylation ( $\mathrm{Li}$ et al. 2019), which suggested that DNA methylation participated in plant drought memory. However, how DNA methylation changes respond to drought and the subsequently drought stress, and how it participated in drought memory is still not clearly identified.

Previously, we established a system that could induce rice drought memory via cycles of mild drought and re-watering treatment and reported 6885 transcripts that involved in the drought memory response. Moreover, we also found some DNA methylation changed located in regions of drought memory genes we found. In this study, we further studied the DNA methylation patterns after cycles of drought stress and re-watering process, and uncovered the contribution of DNA methylation to plant short-term drought memory formation.

\section{Methods}

\section{Plant Materials and Growth Conditions}

Seeds of rice (Oryza sativa L. ssp. Japonica $c v$. Zhonghua 11) were germinated under sterilized condition and placed on four layers of moistened filter paper as described previously ( $\mathrm{Li}$ et al. 2019). Seedlings were grown in darkness for four days at $30^{\circ} \mathrm{C}$ and then transferred to a hydroponic growth system using 1/4 modified Hoagland solution at $28{ }^{\circ} \mathrm{C}$ for 4 weeks. The light/dark cycle was $12 \mathrm{~h} / 12 \mathrm{~h}$ and light intensity was $180 \mu \mathrm{mol} \mathrm{m} \mathrm{m}^{-2} \mathrm{~s}^{-1}$.

\section{Drought and Re-watering Treatment}

4 weeks old seedlings (non-treatment control, R0) were airdried for $80 \mathrm{~min}$ (first drought stress treatment, S1) and fully re-watered for $22 \mathrm{~h}$ (first re-watering samples, R1). To monitor the memory of drought stress, seedlings were sequentially dried and re-watered twice. Leaf samples from R0, S1, R3, and S4 treated seedlings were harvested for analysis (Supplemental Fig. 1). Samples were immediately frozen in liquid nitrogen and stored at $-80^{\circ} \mathrm{C}$ before processing. All the treatments were performed at $28^{\circ} \mathrm{C}$. Relative water content (RWC) was used for rapid estimation of drought stress: $\mathrm{RWC}=(\mathrm{FW}-\mathrm{DW}) /(\mathrm{RW}-\mathrm{DW}) \times 100 \%($ Ding et al. 2014), where FW corresponds to the fresh weight, DW and RW to drought-treated weight and re-watered weight, respectively.

\section{DNA Library Construction and Whole-Genome Bisulfite Sequencing}

Total genomic DNA of rice seedlings were extracted using the CTAB method. The amount and quality of DNA was examined with Nanodrop-2000 (Thermo Fisher Scientific) and agarose gel electrophoresis. DNA library construction and sequencing was performed by Biomarker technologies. In brief, DNA was fragmented via sonication, and then the fragments were ligated with adapters and purified, then bisulfite converted using EpiTech Bisulfite kit (Qiagen, United states). A pair-end sequencing was performed on an illumina Hiseq ${ }^{\mathrm{TM}} 2500$ Platform.

\section{Bioinformatics Analysis}

Raw reads were quality controlled and trimmed and reads were then aligned to the rice reference genome on phytozome (https://phytozome.jgi.doe.gov/pz/portal.html\#!info? alias=Org_Osativa) using BSMAP (version 2.74). The individual methylation ratio was extracted using python from the output of BSMAP mapping results. The methylation rate of 
$\mathrm{mCG}, \mathrm{mCHG}$, and $\mathrm{mCHH}$ in the whole genome was determined as Methylated cytosine/total cytosine. DMRs were calculated by sliding windows with 200 bps slide window width and 50 bps step size. Cytosine sites covered by at least six reads in each bin were considered. Fisher test and False discovery rate (FDR) were used for $P$ value $(P$ value $<0.05)$ calculation and correction. A minimum difference of $40 \%$ methylation level, and also the methylation rate was greater than $10 \%$ in at least one time point was identified as DMRs. For DMRs that only found in the subsequent time points but not the initial one, the threshold of methylation difference for DMRs was set at 30\% for mCG and $\mathrm{mCHG}$, and $20 \%$ for mCHH. Circos software was used to construct Circos Plots (Krzywinski et al. 2009). Short Time-series Expression Miner program (STEM, version 1.3.11) (Ernst and BarJoseph 2006) was used to visualize and compare the methylation rate among each sample to obtain drought memory profiles.

Gene ontology (GO) enrichment analysis was performed using WEGO website (http://wego.genomics.org.cn). Kyoto Encyclopedia of Genes and Genomes (KEGG) pathway enrichment analysis was performed by KEGG orthology based annotation system (KOBAS, version 3.0).

\section{Results}

\section{Differential DNA Methylation in Rice Genome Under Drought Stresses}

According to our previous research, rice seedlings display drought stress memory after cycles of appropriate water deficient treatment. To analyze and visualize the global DNA cytosine methylation pattern after drought stress and the rewatering process, BS-seq was used in this study. Samples were taken from four-week-old rice leaves before (R0) and after the first 2-h drought stress (S1), and also at the time points after the third 22-h re-watering process (R3) and after the fourth drought stress (S4) (Supplemental Fig. 1).

After performing quality control and trimming process, 63-78 millions of high-quality reads were obtained and $88-94 \%$ of them were matched to the rice reference genome with genome coverage of $81.3-91.0 \%$ (Supplemental Table 1). Moreover, all the bisulfite conversion rates were higher than $99.5 \%$, which indicating the accuracy and integrity of whole-genome bisulfite sequencing of this study.

The genome-wide cytosine sites (Supplemental Table 2) and their methylation levels at different time points (R0, S1, R3, S4) under all three sequence contexts (CG, CHG, and $\mathrm{CHH}$; $\mathrm{H}$ represent $\mathrm{A}$, T, or C) were calculated. The results showed that mCG was the most abundant in three contexts in all conditions we tested, while cytosine methylation level under $\mathrm{CHG}$ and $\mathrm{CHH}$ showed comparable levels, which were
$68.56 \%$ and $63.12 \%$ of the numbers under $\mathrm{CG}$ at $\mathrm{R} 0$, respectively (Fig. 1A). The relative DNA methylation rate of $\mathrm{mCG}$ is calculated as $\mathrm{mCG} /(\mathrm{mCG}+\mathrm{mCHG}+\mathrm{mCHH}) \times 100 \%$, the methylation rate of $\mathrm{mCG}$ was $43.18 \%$ at $\mathrm{R} 0$ without any treatment (Fig. 1B), while the genomic $\mathrm{C}$ sites rate under CG sequence context was about $18.96 \%$ in the whole genome (Supplemental Table 2). On the other hand, $\mathrm{mCHG}$ and $\mathrm{mCHH}$ methylation rates were $29.60 \%$ and $27.21 \%$ (Fig. 1B), while $\mathrm{C}$ site rates under $\mathrm{CHG}$ and $\mathrm{CHH}$ in the entire genome were $16.84 \%$ and $61.2 \%$ (Supplemental Table 2), respectively. These results suggested that large numbers of CG sites were cytosine methylated, while only a small part of $\mathrm{CHH}$ sites was methylated even the context of $\mathrm{CHH}$ is most abundant in rice genome.

The genome-wide cytosine methylation levels in each condition (R0, S1, R3, S4) under CG, CHG, and CHH were further compared. DNA methylation level increased after S1 (first drought stress) compare to R0 (without any treatment), among which cytosine methylation levels under CG showed $10.86 \%$ of increasing, while under $\mathrm{CHG}$ and $\mathrm{CHH}$ only $7.52 \%$ and $2.01 \%$ increasing was found compared to their R0, respectively. After subsequently drought stresses at R3, the methylation was reduced to an even lower level compared to R0 (R3 vs R0), which decreased 7.34\%, 5.45\%, and $0.60 \%$ under $\mathrm{CG}, \mathrm{CHG}$, and $\mathrm{CHH}$, respectively. A second induction after subsequent drought stress (S4) was observed under all three sequences, the increase of methylation level (S4 vs R3) was $22.80 \%, 16.87 \%$, and $14.34 \%$ under CG, $\mathrm{CHG}$, and $\mathrm{CHH}$, respectively (Fig. 1A). The corresponding methylation rates were also analyzed, and the results showed that $\mathrm{mCG}$ and $\mathrm{mCHH}$ methylation rate are sensitive to drought stress and re-watering process, while $\mathrm{mCHG}$ rate did not change much (range from 29.01 to $29.62 \%$ ) in all four conditions we tested (Fig. 1B).

To visualize the global pattern in each sample, a heat map on all 12 chromosomes of rice was performed including the information about the DNA methylation levels under different stress stages, the distribution of genes and TEs in all sequence contexts (Fig. 1C). For all the three contexts, the methylated cytosines enriched in the pericentromeric regions where there are low levels of genes in intensity. CHH methylation level was around $10-30 \%$, which was strikingly lower than CG and CHG contexts (Fig. 1C, Supplemental Figure S2), which were about $70-100 \%$ and $10-100 \%$, respectively.

\section{DNA Methylation Involved in Rice Drought Responses and Drought Memory}

DMRs were identified to compare methylation levels after drought stress using sliding-windows approach. Under all contexts of DMRs we detected, CHH DMRs were most abundant (Table 1). We found 44,415 and 45,205 DMRs under $\mathrm{CHH}$ sequence in the first (S1) and subsequent (S4) 

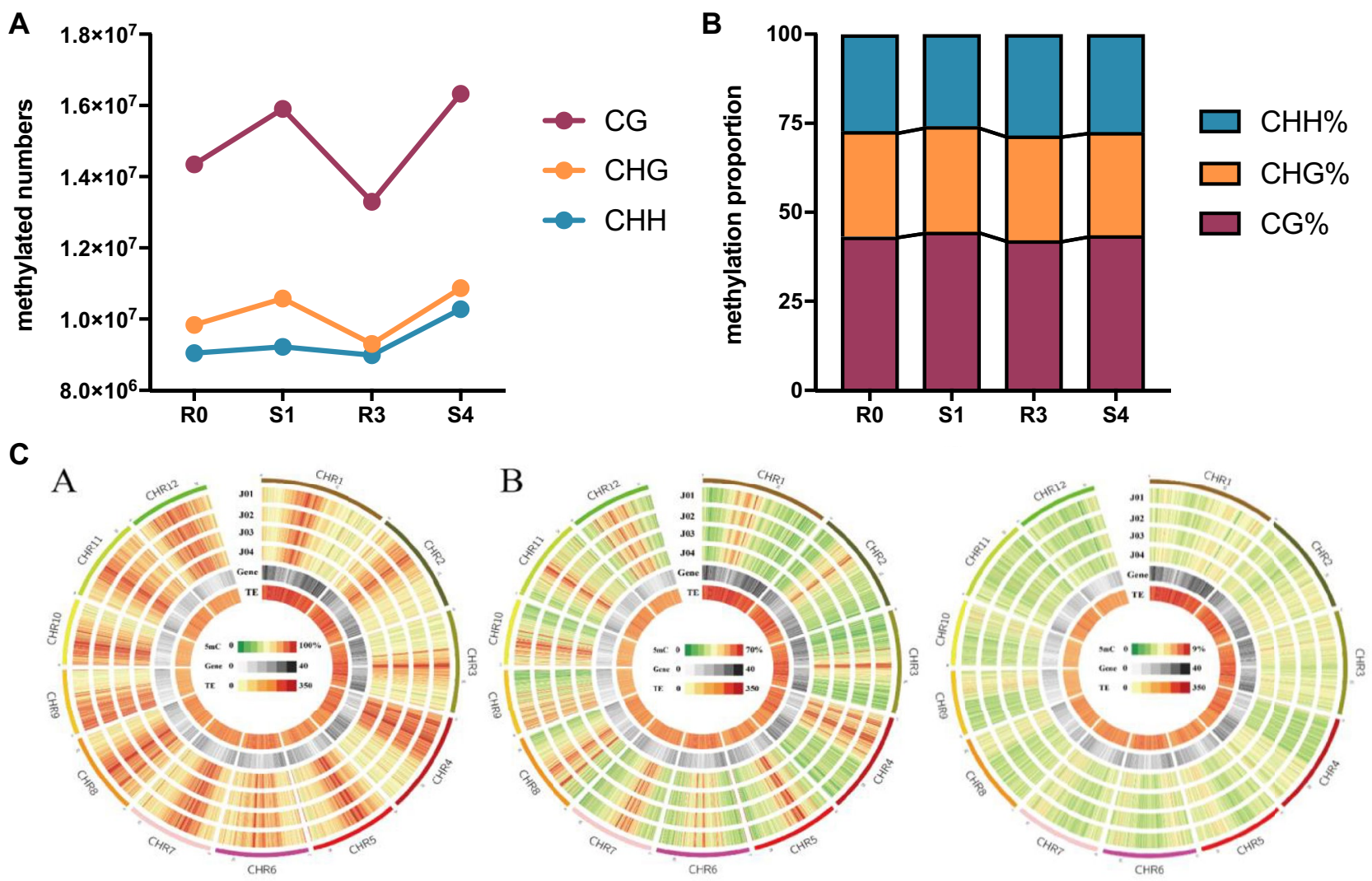

Fig. 1 DNA methylation levels during drought training treatment. A Total number of methylation events under contexts of $\mathrm{CG}, \mathrm{CHG}$, and CGG. B Proportion of methylation under each context. C Circos plots of DNA methylation under contexts of $\mathrm{CG}, \mathrm{CHG}$, and $\mathrm{CHH}$ in chro-

Table 1 Information of DMRs under drought stress

\begin{tabular}{llrrr}
\hline Samples & Type & Hypo* & Hyper* & \multicolumn{1}{c}{ Total } \\
\hline J01_J02 & CG & 4184 & 4883 & 9067 \\
& CHG & 3557 & 5692 & 9249 \\
& CHH & 24,746 & 19,669 & 44,415 \\
J03_J04 & CG & 3289 & 4542 & 7831 \\
& CHG & 2505 & 5633 & 8138 \\
& CHH & 14,817 & 30,388 & 45,205 \\
\hline
\end{tabular}

*The Hypo represents DNA methylation level lower, The Hyper represents DNA methylation level rise

drought stress, while less than 10,000 DMRs under CG (9067 for the first drought stress and 7831 for the second drought stress) or CHG (9249 for the first drought stress and 8138 for the second drought stress) were identified in these two cycles of treatment. Moreover, the number of DMRs under $\mathrm{CHH}$ sequence contexts increased $1.78 \%$ during the subsequence drought treatment (S4) comparing to first drought stress (S1), while the numbers of DMRs under mosomes. Rings of $\mathrm{J} 01, \mathrm{~J} 02$, J03, and $\mathrm{J} 04$ show genomic positions of DNA methylation at R0, S1, R3, and S4 time points. Rings of Gene and TE represent the density of genes and TEs on chromosomes

CG and CHG decreased $13.63 \%$ and $12.01 \%$, respectively (Table 1). The number of both hypermethylation and hypomethylation in CG and CHG DMRs were decreased in subsequent drought stress (S4) than first stress treatment (S1), although we found that most of the regions in genome of DNA methylation changes were different (Table 1). Interestingly, the number of hypermethylation in CHH DMRs was increased from 19,669 to 39,388 (54.50\%) dramatically. This result suggests that cytosine methylation participated in rice short-term drought memory and $\mathrm{CHH}$ methylation is very sensitive to drought stress.

Next, we examined the distribution of DMRs in genomic features such as TEs, 2-kb upstream of genes (upstream), gene bodies (body), 2-kb downstream of genes (downstream), and intergenic regions (IG) (Fig. 2). Most CGrelated DMRs were located at IG and 2-kb upstream (promoter region) of genes, while most CHG- and CHHrelated DMRs were located in TE region and IG. Overall, more hypermethylation were found than hypomethylation were found in both R0-S1 and R3-S4, regardless CG-, CHG-, or CHH-related DMRs. Comparing R0-S1 and 

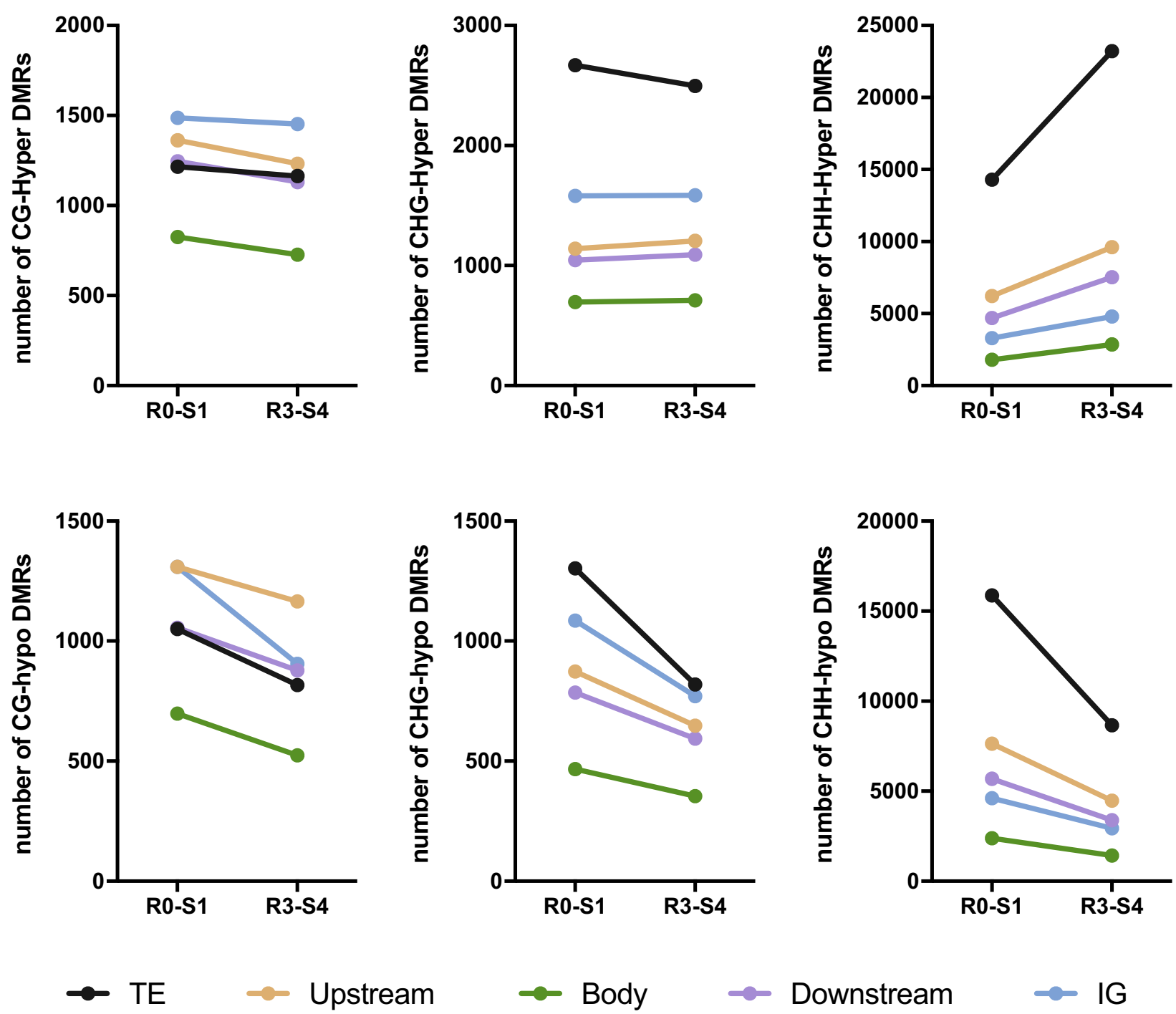

Fig. 2 Distribution of drought DMRs under initial and subsequently drought stresses. A Numbers of hypermethylation DMRs under the contexts of CG, CHG, and CHH among TE, 2-kbs upstream of a gene, gene body, and 2-kbs downstream of a gene, and IGs under

R3-S4, CHH-related hypermethylation increased dramatically $(54.50 \%)$, which TE located DMRs contributed the most $(83.11 \%$ of the total). On the contrary, in CG- and CHG-related hypermethylation, TE located DMRs decreased slightly (4.28\% and 6.45\%) (Fig. 2). Less hypomethylated DMRs were found in subsequent drought stress than the first drought treatment regardless sequence contexts, with $21.39 \%, 29.58 \%$, and $40.12 \%$ decrease under CG, CHG, and $\mathrm{CHH}$, respectively (Fig. 2). Meanwhile, hypermethylated DMRs showed complicated patterns. These results showed that CHG-related methylation did not respond much comparing to CG- and CHH-related hypermethylation. Most interestingly, TE located methylation was found to be most sensitive in responding to the sequential drought stress (Fig. 2). two drought stresses. B Numbers of hypomethylation DMRs under the contexts of CG, CHG, and CHH among TE, 2-kbs upstream of a gene, gene body, and 2-kbs downstream of a gene, and IGs under two drought stresses

To better compare the DNA methylation under the initial and subsequently drought responses, we performed GO analyses of DMR-related genes obtained from both treatments. In total, 22,605 and 22,506 DMR-related genes were identified in the first and the subsequent drought stress, respectively. DMR-related genes were categorized into three groups: biological process, cellular component, and molecular function. Our results showed that in both stress cycles, DMR-related genes were associated with catalytic activity, component binding, metabolic process, cellular process, and other processes (Supplemental Fig. S3). Notably, although we did not observe a significant difference between two drought stress treatments in GO analysis, a lot of DMRrelated genes enrich in membrane-bounded organelle and in 
biological process of response to stimulus including abiotic stimulus.

\section{Rice Drought Stress Memory-Related DMRs Showed Dynamic and Distinct Patterns}

Previously, we defined memory genes as those transcript levels in subsequent stress were significantly different from their levels during the initial drought stress period. Among 10,124 memory genes we identified, 5373 memory transcripts were identified to be possibly regulated by DNA methylation ( $\mathrm{Li}$ et al. 2019). To further explore DMRs patterns in rice related to drought stress memory, we defined memory DMRs based on the following criteria: among regions that were responsive to the first drought stress in genome, those regions for which DNA methylation levels in subsequent stress periods were significantly different from their levels during the first stress period (R0-S1). STEM program was utilized to cluster the memory DMRs according their methylation status. In all, 10 out of 26 clusters of DMRs represented memory DMRs (Profile 0, 1, 3, 4, $7,18,21,22,24,25)$, which memory patterns were classified as lineage, stable, accumulated, dosage dependent, and Initial memory (Fig. 3A). The rest DMRs do not follow the memory patterns which identified in R0-S1, R0-R3, R0-R4 were classified as non-memory DMRs.

Our results showed that $27.73 \%$ and $26 \%$ DMRs under sequence contexts $\mathrm{CG}$ and $\mathrm{CHH}$ were clustered as memory DMRs, respectively. While only about $22.82 \%$ DMRs under sequence contexts CHG were memory DMRs. Most of memory DMRs gathered in profile4, profile7, profile18, and profile21 (Fig. 3B), which indicated that memory DMRs initially changed (both hypomethylation and hypermethylation) in R0-S1 stage, maintained at a stable level despite subsequent drought treatment (R3-S4). We further examined the distribution of memory DMRs, comparing with non-memory DMRs identified in genomic features. In memory MDRs under CHG context, TE-related distribution is lower than in non-memory DMRs (Fig. 4A), with $21.63 \%$ in memory DMRs and $34.29 \%$ in non-memory DMRs. Meanwhile, the distribution of DMRs under CHG contexts showed similar pattern related to gene body, upstream, downstream, and IG region between memory (16.45\%) and non-memory DMRs (15.22\%) (Fig. 4B). Interestingly, we also noticed that under contexts of $\mathrm{CG}$ and $\mathrm{CHH}$ located at IG, the proportion of memory DMRs were $12.45 \%$ and $36.84 \%$ higher than nonmemory DMRs, while the proportion of upstream and downstream of genes were less $(8.70 \%$ and $14.02 \%$ less under CG and $\mathrm{CHH}$ upstream of genes, $10.11 \%$ and $7.20 \%$ less under $\mathrm{CG}$ and $\mathrm{CHH}$ downstream of genes) (Fig. 4B).

Distribution of TEs over DMRs was examined and the distances of DMRs between their nearest TEs were analyzed too. We found the overlap rates (DMRs overlap with TEs) were comparable between CG-related memory DMRs and non-memory DMRs, while the rates in CHG- and $\mathrm{CHH}$ related non-memory DMRs showed higher ratios compare to their memory DMRs, respectively ( $38.1 \%$ in memory vs $39.3 \%$ in non-memory under CG contexts, $43.9 \%$ in memory vs $49.2 \%$ in non-memory under CHG contexts, and $67.2 \%$ vs $74.6 \%$ under $\mathrm{CHH}$ contexts). It is also noteworthy that there are significant differences between memory DMRs and non-memory DMRs regarding distances to TE under all sequence contexts, as peaks shifted noticeably in density plots comparing the distance between memory/non-memory DMRs and TEs (Fig. 4C). Distances between DMRs and TEs were found globally smaller in memory DMRs which suggested that memory DMR might affect TEs globally in drought memory.

GO enrichment and KEGG were performed comparing the memory and non-memory DMR-related genes to obtain a better idea of drought memory mechanism. Similar patterns of GO enrichment were found in both memory DMRs and non-memory DMRs (Supplemental Figure S4). Results of KEGG enrichment showed that both memory DMRs and non-memory DMRs regulated stilbenoid, diarylheptanoid, and gingerol biosynthesis, biosynthesis of secondary metabolites, metabolic pathways, limonene, and pinene degradation pathways (Fig. 5). Interestingly, we found alphaLinolenic acid metabolism, linoleic acid metabolism, biosynthesis of amino acids, Glycerophospholipid metabolism, Cysteine and methionine metabolism, and Lysine biosynthesis pathways were regulated significantly by memory DMRs specifically, while non-memory DMRs specifically regulated phenylpropanoid biosynthesis, starch and sucrose metabolism, zeatin biosynthesis, phosphatidylinostiol signaling system, diterpendoid biosynthesis, and plant-pathogen interaction pathways. Overall, our results suggested that memory DMRs may play important roles in drought memory and regulate pathways for plant coping with repeated drought stress.

\section{Memory DMRs Could Directly Regulate Rice Drought Memory Genes}

Our previous results showed that 5373 drought memory transcripts might be regulated by DNA methylation by linkage analysis on differentially DNA methylated regions and expression of memory genes ( $\mathrm{Li}$ et al. 2019). The gene encoding the proline biosynthetic enzyme $\Delta 1$-pyrroline-5carboxylate synthetase 1 (P5CS1) was reported to be a critical gene in rice drought stress memory (Li et al. 2019). The expression of two P5CS1 homologous (LOC_Os01g62900 and LOC_Os05g38150) were found rapidly induced after the first drought stress (S1) and reach a peak at R2, then remained stable throughout the subsequent drought stress treatment, in correspond with the level of free proline 


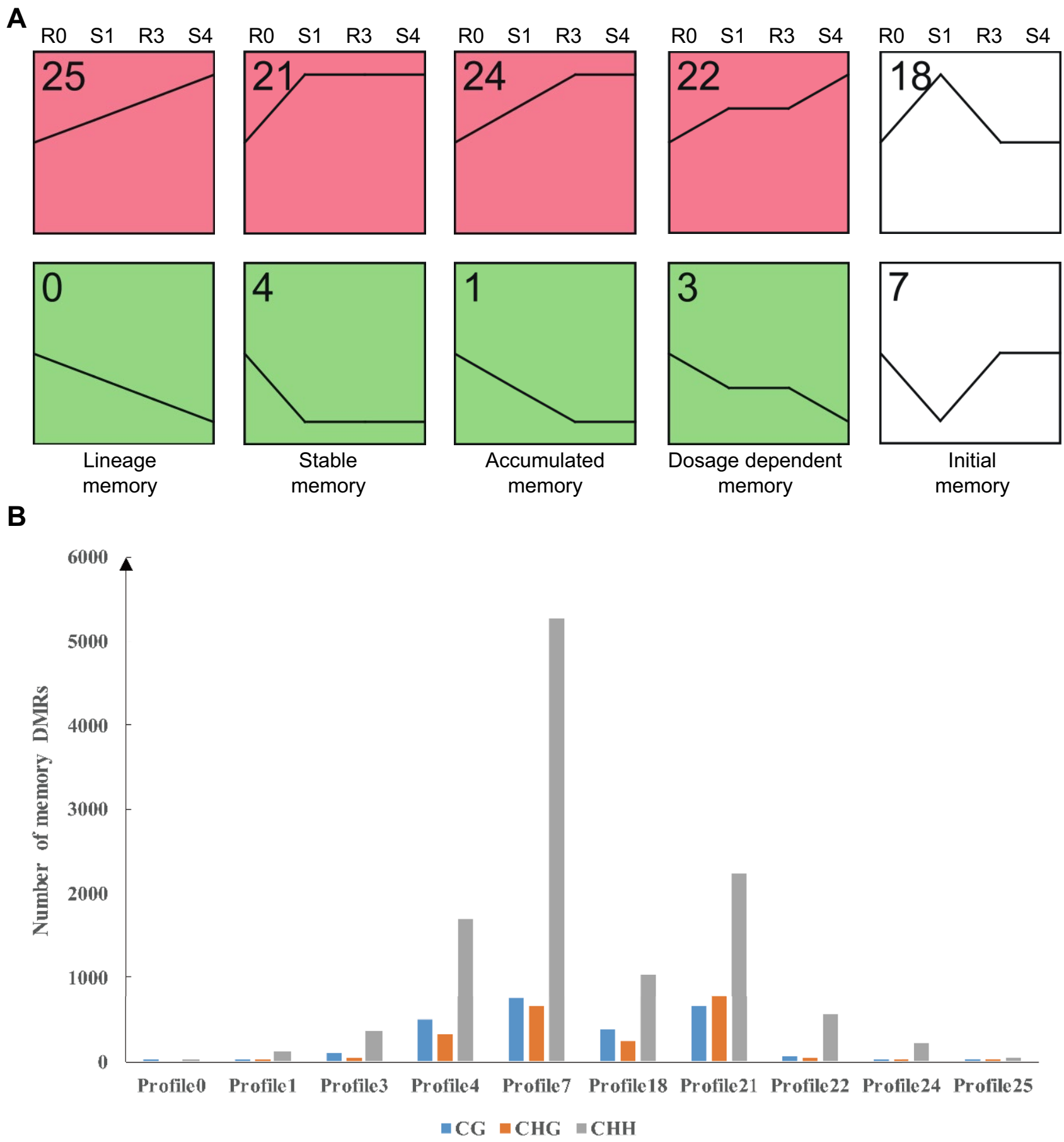

Fig. 3 Drought-Memory DMR profiles in rice. A CG methylation drought-memory DMR profiles under different drought and re-watering treatment stages. B Distribution of memory DMRs in each profile

content( $\mathrm{Li}$ et al. 2019). In the current study, the methylation status of these two drought memory P5CS1 genes in rice were explored to test if the drought memory genes were directly regulated by memory DMRs. Our results showed that memory DMRs were located in promoter region of LOC_Os05g38150 and in gene body of LOC_Os01g62900, and both the memory DMR pattern belonged to profile 21 (Figs. 3A and 6A).

Although DNA methylation was found involved in drought memory genes in our previous work ( $\mathrm{Li}$ et al. 2019), the relationship between memory genes and memory DMRs was not known. Therefore, we analyzed 663 


\section{A

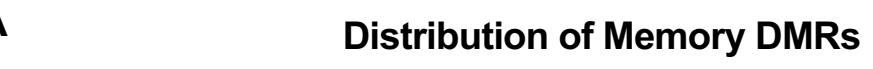

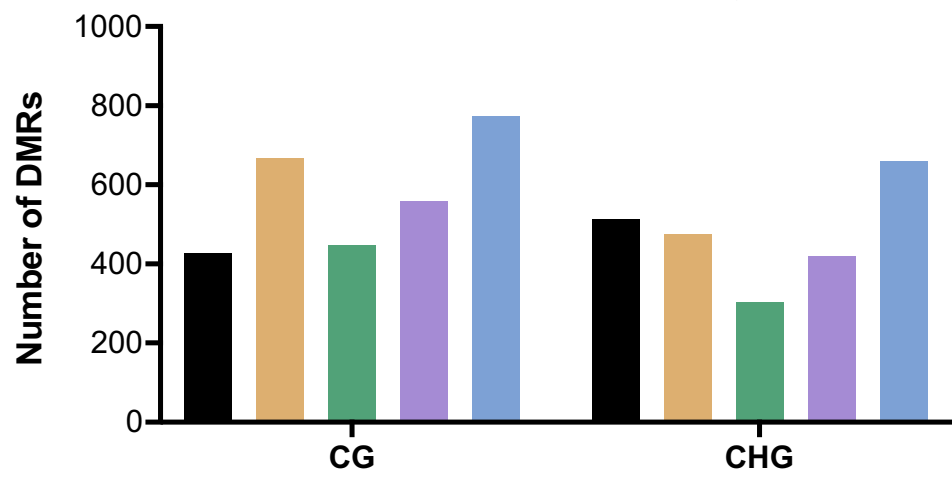
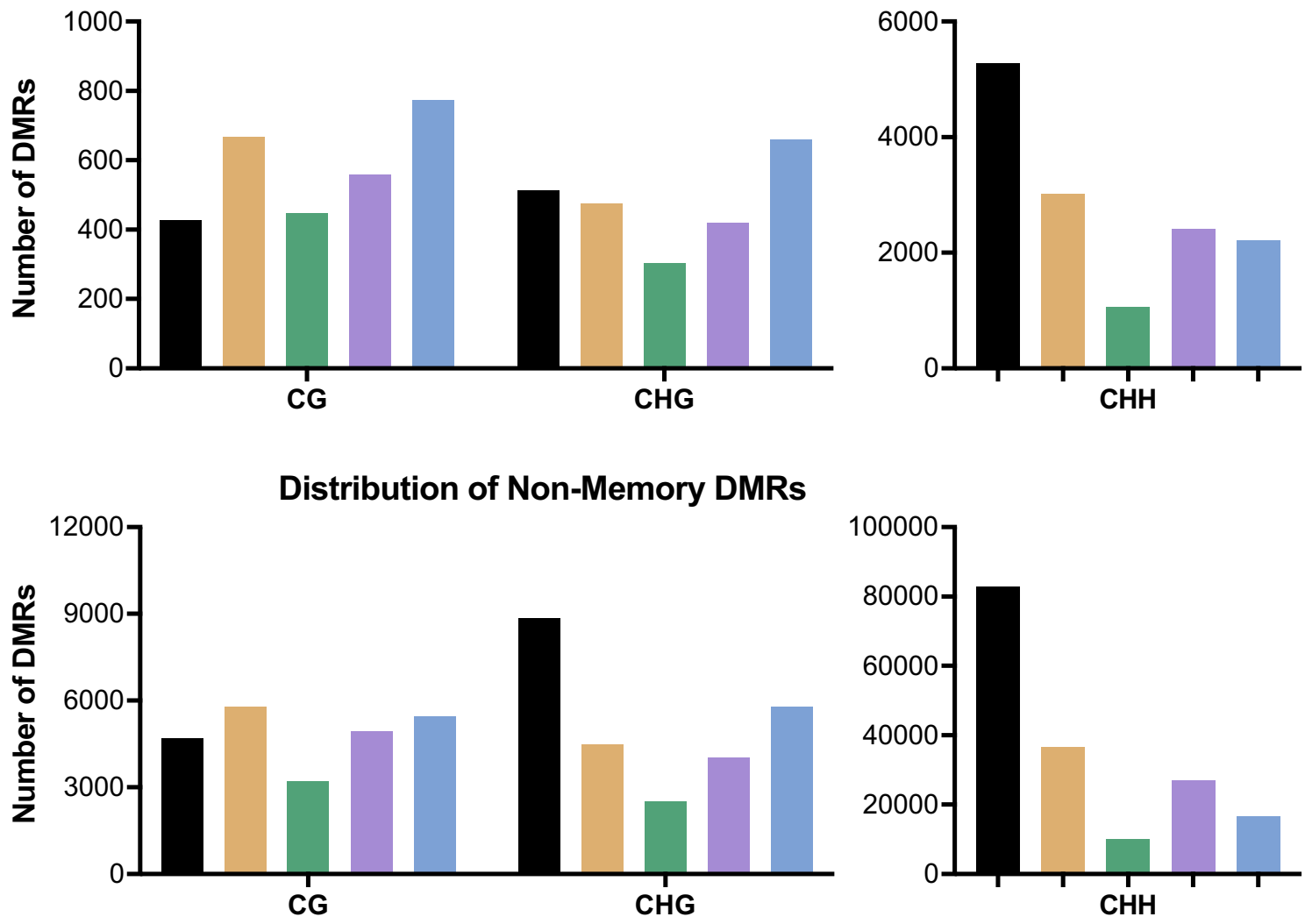

- TE Upstream

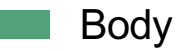

Downstream

IG

B

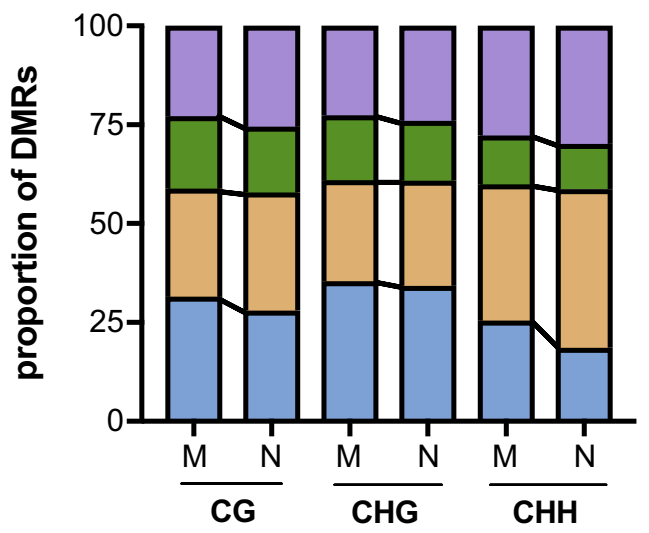

Fig. 4 Drought-memory DMRs distinct from drought non-memory DMRs. A Distribution of drought memory and drought non-memory DMRs under the contexts of $\mathrm{CG}, \mathrm{CHG}$, and $\mathrm{CHH}$ among TE, 2-kbs upstream of a gene, gene body, and 2-kbs downstream of a gene, and IGs. B Proportion of DMRs identified in (A) under each context. $M$

drought memory genes which identified to have memory DMRs distribution in gene body region or $2 \mathrm{~kb}$ flanking region of the gene. The numbers of memory DMRs under

C

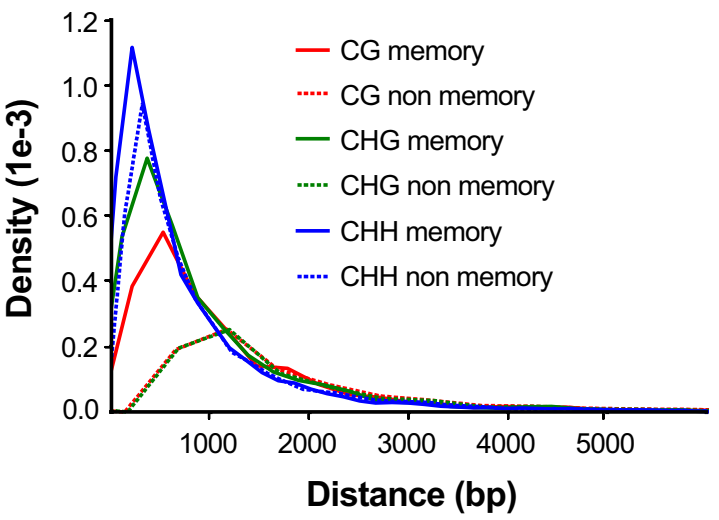

memory DMRs; $N$ non-memory DMRs. Colors represent DMRs located in 2-kb upstream of a gene, gene body, 2-kb downstream of a gene, or IGs. C Density map of the distances of memory DMRs and non-memory DMRs to TEs (Color figure online)

the sequence contexts $\mathrm{CG}, \mathrm{CHG}$, and $\mathrm{CHH}$ were found to be 151,94 , and 484 among these drought memory genes, respectively. Next, we performed GO enrichment and KEGG 


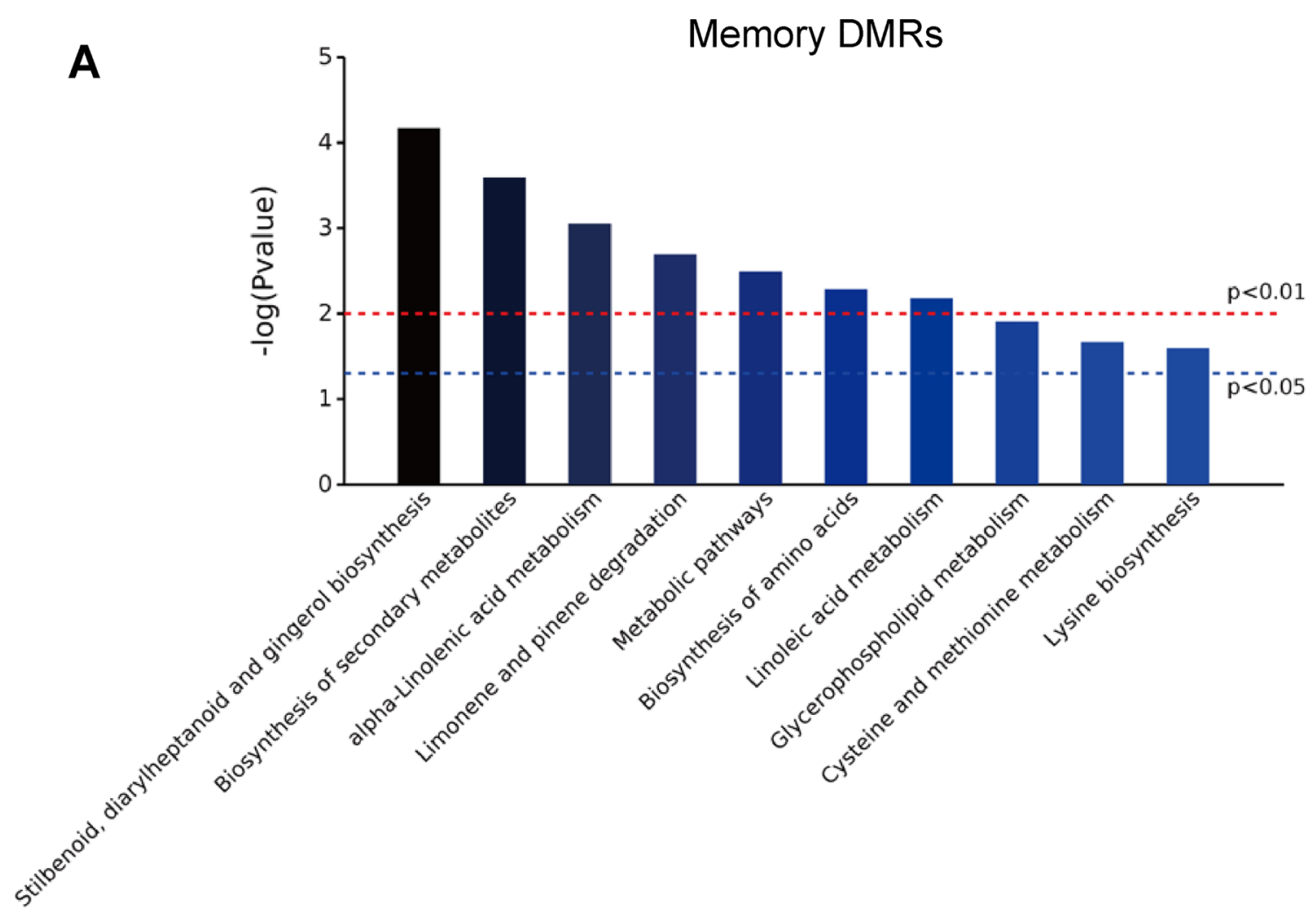

B

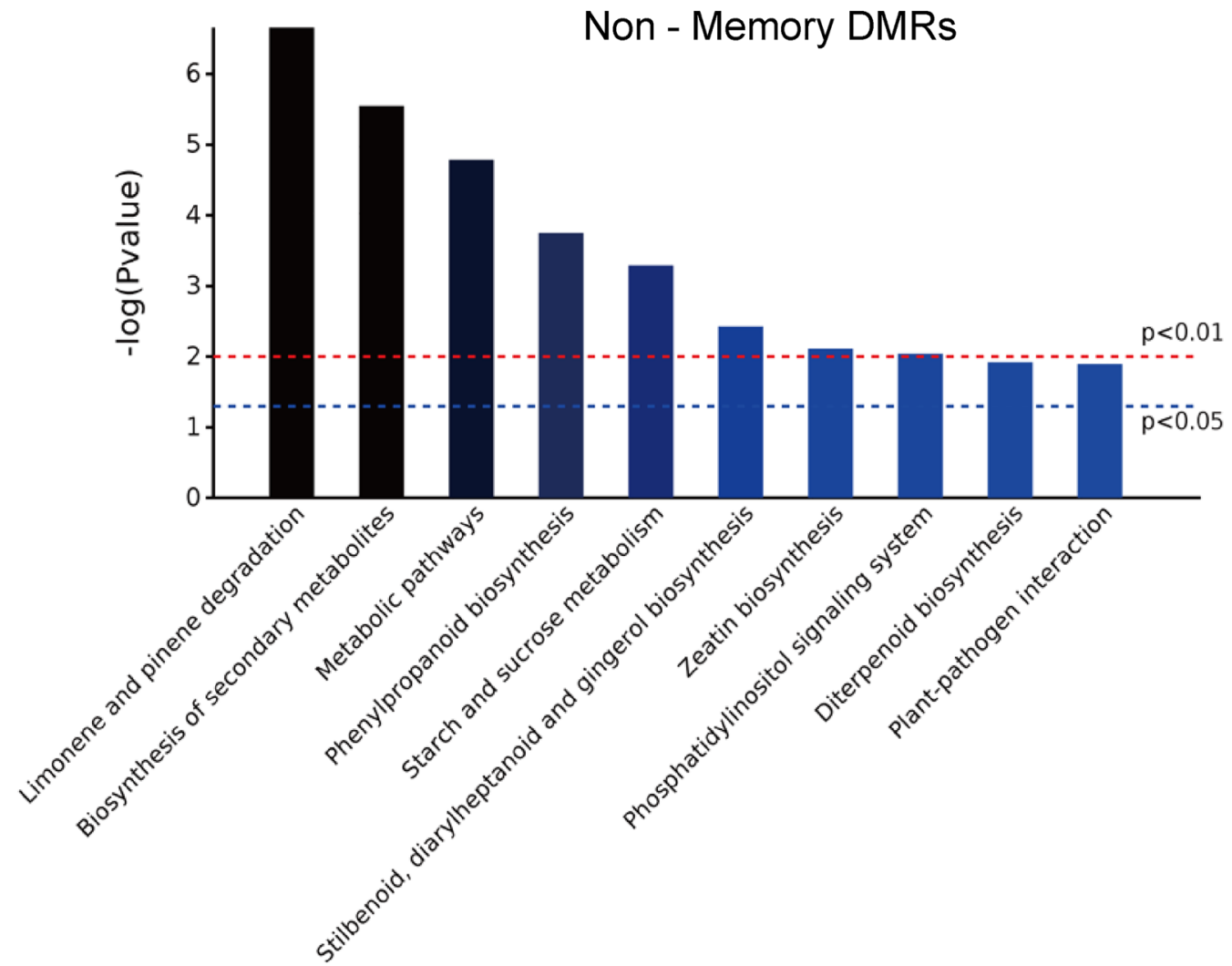

Fig. 5 KEGG enrichment analysis of DMR-related genes under recurrent drought stresses. A KEGG enrichment analysis of drought-memory DMRs-related genes. B KEGG enrichment of non-memory DMRs-related genes 
pathway analysis of these 663 genes to have a better idea of how these drought-memory DMRs regulate memory genes (Supplemental Fig. S5 and Fig. 6B). Most of the memory genes were found involving in metabolic, cellular process, binding, and catalytic activity. Results of KEGG enrichment analysis showed that memory DMRs related memory genes mainly through sesquiterpenoid and triterpenoid biosynthesis, phenylpropanoid biosynthesis, arginine and proline metabolism pathways. These evidences supported memory DMRs involved in rice drought memory by directly regulate rice drought memory genes.

\section{Discussion}

Growing evidence of DNA methylation play important roles in rice response to drought has been reported in recent years. However, how DNA methylation involved in drought memory is still unclear. In this study, we assessed the dynamic patterns of DNA methylation patterns in rice cultivar under recurrent drought stresses and recovery treatments using genome-wide bisulphite sequencing at a single base resolution methylome profiling level. This method enables us to observe and explain how DNA methylation change dynamically to regulate rice response to the drought stresses that happens repeatedly in a memory way.

Different plant species may have diverse methylation patterns that occur under the contexts of $\mathrm{CG}, \mathrm{CHG}$, and $\mathrm{CHH}$. In our results, most of CG sequences were methylated $(80-100 \%)$ while only a small portion of $\mathrm{CHH}$ sequences were methylated (10-30\%) of the genome. The methylation rate of $\mathrm{mCG}$ was $43.18 \%$, and $\mathrm{mCHG}$ and $\mathrm{mCHH}$ methylation rates were $29.60 \%$ and $27.21 \%$ at R0 without any treatment (Fig. 1B). This result is similar with previous study showed that the percentage of methylcytosines was 46.70-49.52\%, 28.36-30.72\%, and 19.76-24.30\% under CG, CHG, and CHH context (Rajkumar et al. 2020). The slight differences might because the differences in rice cultivar, development stages, growth, and stress treatment conditions. Similar levels of methylation were observed in forward and reverse strands under all contexts in all the samples, which are consistent with a previous report (Garg et al. 2015).

Under drought stress, many previous studies reported DNA methylation level decreased or not change (Rajkumar et al. 2020; Wang et al. 2011; Zheng et al. 2013), while other studies reported strongly increased when rice seedlings encountered drought stress (Gayacharan 2013; Xia et al. 2017). In our study, we observed the general methylation level increased in response to drought stress (R0-S1), which in coincidence with the latter reports. Furthermore, we also observed that the methylation level increased again in the subsequently drought stress (R3-S4) when the methylation level recovered after the initial drought stress. This indicates that the methylation status of rice in responses to drought stress is high dynamic throughout the duration of stress and the recovery time, and also may vary among different rice genotypes growing/stressing under different conditions. This is also supported by another study showed that the methylome divergence among the cultivars is higher than it changes under drought stress within one cultivar (Rajkumar et al. 2020). Our results also suggested that methylation under $\mathrm{CG}$ and $\mathrm{CHH}$ contexts are more sensitive and responsive to drought stress than that under $\mathrm{CHG}$ context.

DMRs in response to drought stress were used to study the methylation dynamics. In rice, more DMRs in sensitive genotype than in tolerant line, and more hypermethylated DMRs were reported compare hypomethylated ones in both drought-sensitive and drought-tolerant lines under drought stress (Wang et al. 2016). This was also observed in our line (Zhonghua 11), which is a drought-sensitive genotype. In our results, much higher numbers of DMRs were identified under $\mathrm{CHH}$ context, which confirmed the important role of $\mathrm{CHH}$ methylation under drought stress as reported previously (Rajkumar et al. 2020). DMRs identified in our report was significantly higher than the previous reports, maybe due to the cultivar and the way of detecting its methylation status and data analysis (whole-genome bisulphite sequencing vs. MSAPs, MeDIP-seq), also might because that the time points before and after both stress treatments were included in our study. Our results shown that subsequently drought stress (S4) resulted less hypomethylated DMRs compare to the initial drought treatment under contexts of all sequences (CG, $\mathrm{CHG}$, and $\mathrm{CHH}$ ), regardless of their locations (TEs, gene body, or Igs). These results suggested that hypomethylation is more stable in constantly drought stressed plant. More interestingly, the hypermethylated DMRs in our study showed different patterns: with increasing numbers after subsequently drought stress under $\mathrm{CHH}$ context while the numbers of hypermethylated DMRs under CG and CHG were only slightly decreased or stable. Together with previous report showed that more DMRs under $\mathrm{CHH}$ context were found in response to drought (Rajkumar et al. 2020), our results confirmed that methylation occur under different contexts were differentially influenced by constantly drought stress, and CHH-related DNA methylation might affect TEs globally, and take important roles in drought memory.

Although drought memory genes in rice were well described, the mechanism on how they were regulated still needs to be explored and drought-memory DMRs were rarely studied (Auler et al. 2017; Li et al. 2019; Liu et al. 2016). In our study, drought stress DMRs showed dynamic and distinctive patterns when treat with recurrent drought stresses. We also defined patterns of memory DMRs to better reflect the ability and effects of DNA methylation on rice drought memory, 10 out of 26 clusters of DMRs were identified as memory DMRs (Profile 0, 1, 3, 4, 7, 18, 21, 22, 
A

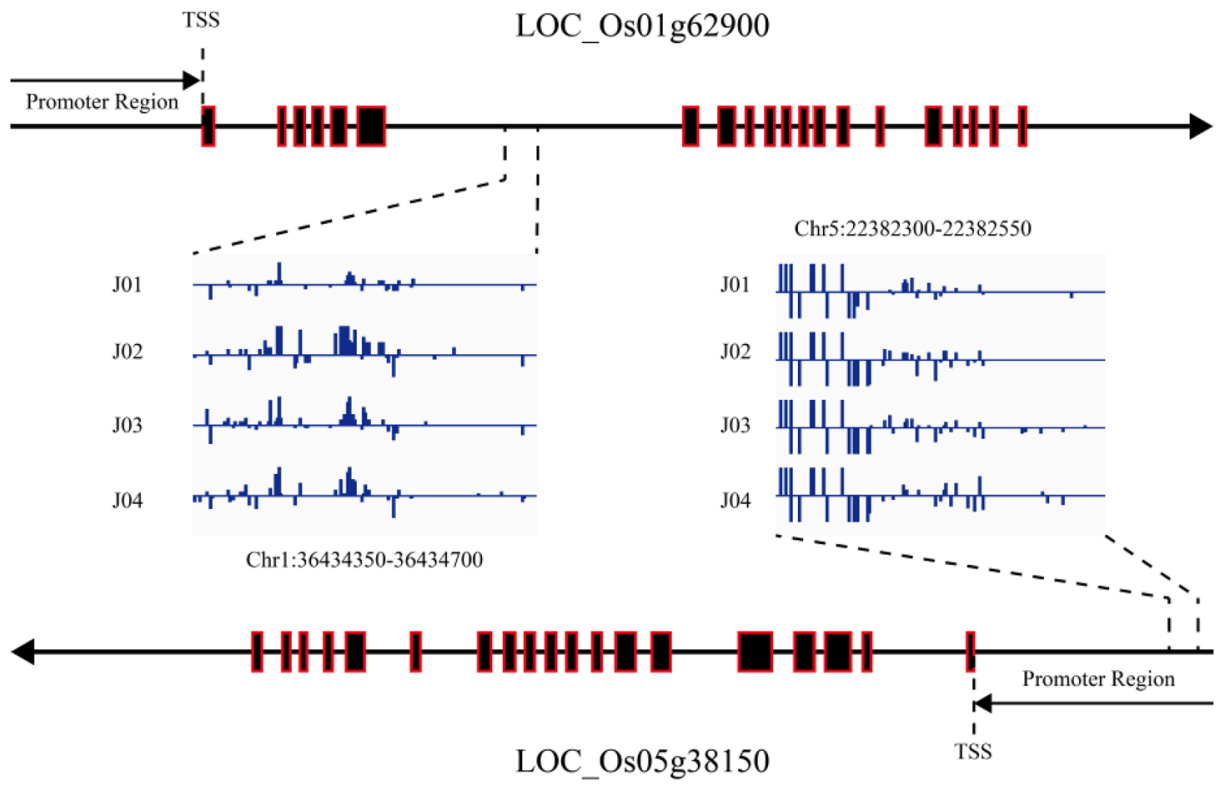

B

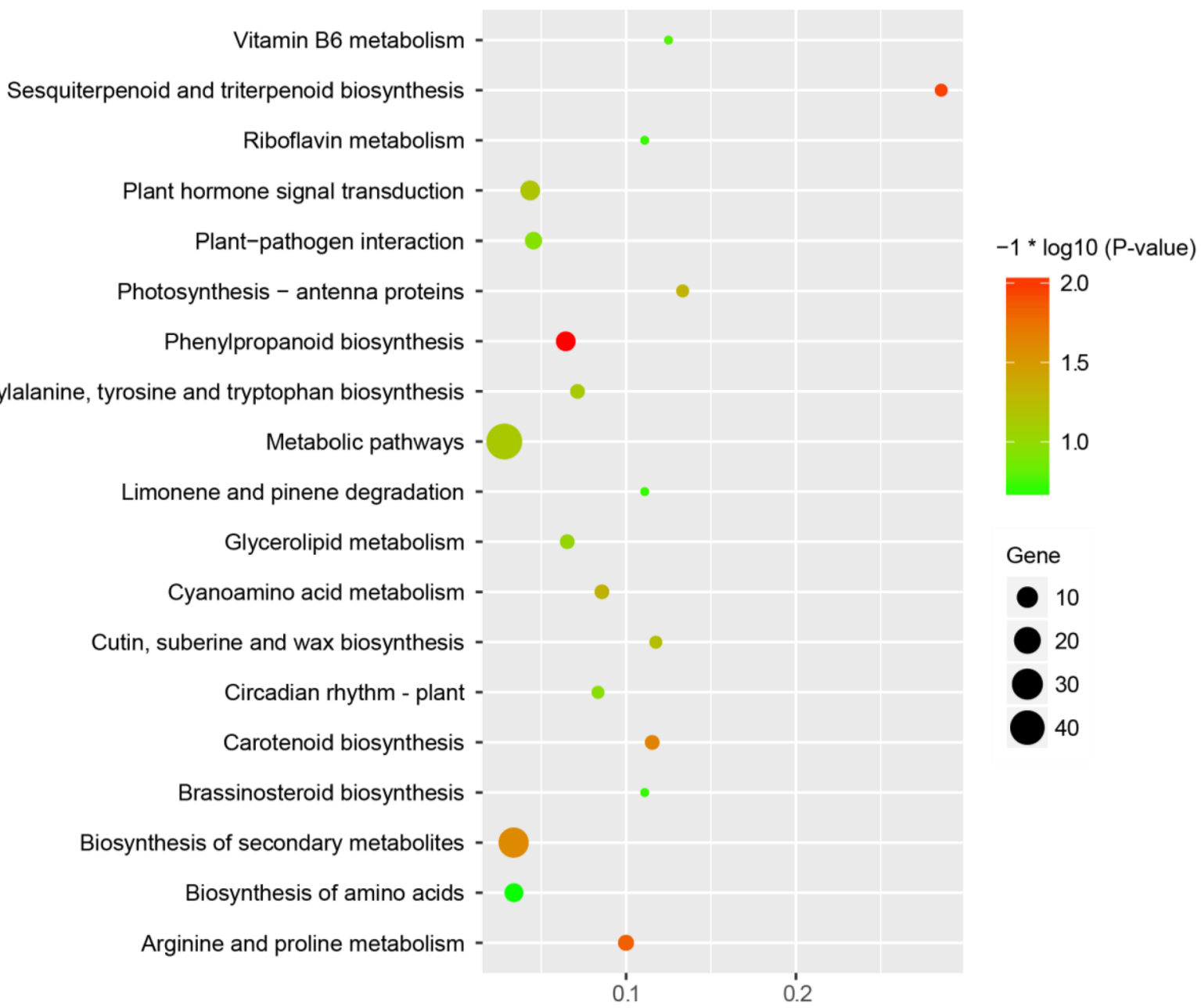

Rich Factor 
४Fig. 6 Drought-memory DMRs directly regulate drought memory genes. A Genome browser views of DMRs in two homologous genes of P5CS1 (LOC_Os05g38150 and LOC_Os01g62900). B KEGG enrichment analysis of memory genes regulated by memory DMRs. The size of each point indicates numbers of genes. Color indicates the range of $P$ values. Rich Factor indicates the ratio of the number of memory genes regulated by memory DMRs in the pathway relative to all genes in these pathways (Color figure online)

$24,25)$, according to their methylated status which classified as lineage, stable, accumulated, dosage dependent, and initial memory patterns. It is known that methylation occurs at diverse sites could affect different changes in gene regulation. Methylations in promoter regions are generally considered negatively associated with gene expression, while DNA methylation on genes body can cause both silencing or activation of genes. Meanwhile, methylation located on Igs may regulate alternative or antisense transcription. In our results, most DMRs were found targeted to TEs and least were targeted to gene bodies, which showed the important roles of DMRs on TEs to regulate drought memory. Comparing memory DMRs with non-memory DMRs, similar distribution patterns were found under the $\mathrm{CG}, \mathrm{CHG}$, and $\mathrm{CHG}$ contexts. However, distances of drought-memory DMRs to TEs were found significantly different when comparing their target sites. Under all context, the distances between memory DMRs to TEs were found significantly smaller than non-memory DMRs, with CHH-related ones showed the nearest comparing with CG and CHG. Similar distribution patterns were found between CG and CHG related Nonmemory DMRs, while CHH-related DMRs distributed much nearer. Our results revealed the close relationship of TEs and drought-memory DMRs, and support the idea that epigenetic factors play a crucial role in drought memory formation. It would be interesting to reveal how they specifically regulate the methylation in drought memory in the future as $\mathrm{CHH}$ sites are governed by DRM1/2 and CMT2.

Previously, we detected 6885 transcripts involved in drought memory response during recurring drought stresses. Other than transcriptional memory, short-term epigenetic memory was discovered in the current report. GO terms and KEGG pathway analysis of the memory DMRs- and non-memory DMRs-related genes showed that they enriched in the same pathway biosynthesis of secondary metabolites, metabolic pathways, limonene and pinene degradation pathways, while memory DMRs specifically enriched in alpha-Linolenic acid metabolism, linoleic acid metabolism, biosynthesis of amino acids, Glycerophospholipid metabolism, Cysteine and methionine metabolism, and Lysine biosynthesis pathways. Previously, we found that 5373 memory transcripts might be regulated by DNA methylation. In this report, we cross checked the memory DMRs-related genes and find 663 drought memory genes have memory DMRs distribution, which enriched in sesquiterpeoid and triterpenoid biosynthesis, phenylpropanoid biosynthesis, arginine and proline metabolism pathways. The two proline biosynthetic enzymes P5CS1 in rice also gave solid evidence that memory DMRs could directly regulate rice drought memory genes. These results further confirmed that shortterm epigenetic memory DNA methylation participated in rice seedlings drought stress memory by regulating genes, in addition to affecting TEs. Epigenetic memory is known could inherit and transmit to subsequent generations after exposed to drought stress conditions and increase their tolerance to drought and survival rate. How is the relationship between short-term DNA methylation memory and epigenetic memory which inherited to the next generations? How DNA methylation and histone acetylation work in concert to regulate memory formation? Those would be interesting questions for further studies.

\section{Conclusion}

Sessile plants experience constant drought challenges throughout their lives. Acute responses to drought stress have been studied worldwide, however, the drought memory mechanisms which are critical for plant coping with recurring and repetitive drought stress are less well understood. Through genome-wide bisulphite sequencing, our study provided new insight on how DNA methylation dynamic changed and showed that short-term epigenetic memory probably regulated the global expression of genes and TEs in rice drought memory.

Supplementary Information The online version contains supplementary material available at https://doi.org/10.1007/s00344-021-10483-3.

Acknowledgements This work was supported by the National Natural Science Foundation of China (31971410), National Science and Technology Plan (2014AA10604-16), Major Projects of Yunnan Science and Technology (202102AE090004), Yunnan Science Foundation (202001AT070361), Personnel Training Program of Yunnan Technical Innovation (2018HB113), Outstanding Young Scholar Program and Yunling Scholar Program of Yunnan Province and Yunnan Rice Product System (KJTX001).

Author Contributions SYK, PH, and GJL acquired the data, GJL and LD analyzed data, HHL, ZGW, and WHL pretreated materials, LD wrote the manuscript; LL, QYG, and PRY designed the study, LL wrote the manuscript and revised the manuscript.

Data Availability Methylome data have been deposited in Genome Sequence Archive (GSA), http://bigd.big.ac.cn/gsub/submit/gsa/subCR A001313/contents. 


\section{Declarations}

Conflict of interest The authors declare no competing interests.

Open Access This article is licensed under a Creative Commons Attribution 4.0 International License, which permits use, sharing, adaptation, distribution and reproduction in any medium or format, as long as you give appropriate credit to the original author(s) and the source, provide a link to the Creative Commons licence, and indicate if changes were made. The images or other third party material in this article are included in the article's Creative Commons licence, unless indicated otherwise in a credit line to the material. If material is not included in the article's Creative Commons licence and your intended use is not permitted by statutory regulation or exceeds the permitted use, you will need to obtain permission directly from the copyright holder. To view a copy of this licence, visit http://creativecommons.org/licenses/by/4.0/.

\section{References}

Alves RDFB, Menezes-Silva PE, Sousa LF, Loram-Lourenço L, Silva MLF, Almeida SES, Silva FG, Perez de Souza L, Fernie AR, Farnese FS (2020) Evidence of drought memory in Dipteryx alata indicates differential acclimation of plants to savanna conditions. Sci Rep 10(1):16455. https://doi.org/10.1038/ s41598-020-73423-3

Auler PA, do Amaral MN, dos Rodrigues GS, Benitez LC, da Maia LC, Souza GM, Braga EJB (2017) Molecular responses to recurrent drought in two contrasting rice genotypes. Planta 246(5):899-914. https://doi.org/10.1007/s00425-017-2736-2

Ding ZJ, Yan JY, Xu XY, Yu DQ, Li GX, Zhang SQ, Zheng SJ (2014) Transcription factor WRKY46 regulates osmotic stress responses and stomatal movement independently in Arabidopsis. Plant $\mathbf{J}$ 79(1):13-27. https://doi.org/10.1111/tpj.12538

Domb K, Katz A, Harris K, Yaari R, Kaisler E, Nguyen V, Hong U, Griess O, Heskiau KG, Ohad N, Zemach A (2020) DNA methylation mutants in Physcomitrella patens elucidate individual roles of CG and non-CG methylation in genome regulation. Proc Natl Acad Sci USA 117(52):33700-33710. https://doi.org/10.1073/ pnas.2011361117

Ernst J, Bar-Joseph Z (2006) STEM: a tool for the analysis of short time series gene expression data. BMC Bioinform 7(1):191. https://doi. org/10.1186/1471-2105-7-191

Fàbregas N, Fernie AR (2019) The metabolic response to drought. J Exp Bot 70(4):1077-1085. https://doi.org/10.1093/jxb/ery437

Fernández de Simón B, Sanz M, Sánchez-Gómez D, Cadahía E, Aranda I (2020) Rising $\left[\mathrm{CO}_{2}\right]$ effect on leaf drought-induced metabolome in Pinus pinaster Aiton: ontogenetic- and genotypic-specific response exhibit different metabolic strategies. Plant Physiol Biochem 149:201-216. https://doi.org/10.1016/j.plaphy.2020.02.011

Ganie SA, Ahammed GJ (2021) Dynamics of cell wall structure and related genomic resources for drought tolerance in rice. Plant Cell Rep 40(3):437-459. https://doi.org/10.1007/s00299-020-02649-2

Garg R, Narayana Chevala V, Shankar R, Jain M (2015) Divergent DNA methylation patterns associated with gene expression in rice cultivars with contrasting drought and salinity stress response. Sci Rep 5(1):14922. https://doi.org/10.1038/srep14922

Gayacharan JAJ (2013) Epigenetic responses to drought stress in rice (Oryza sativa L.). Physiol Mol Biol Plants 19(3):379-387. https:// doi.org/10.1007/s12298-013-0176-4

Godwin J, Farrona S (2020) Plant epigenetic stress memory induced by drought: a physiological and molecular perspective. Methods Mol Biol 2093:243-259. https://doi.org/10.1007/978-1-07160179-2 17
Hu H, Dai M, Yao J, Xiao B, Li X, Zhang Q, Xiong L (2006) Overexpressing a NAM, ATAF, and CUC (NAC) transcription factor enhances drought resistance and salt tolerance in rice. Proc Natl Acad Sci 103(35):12987-12992. https://doi.org/10.1073/pnas. 0604882103

Hu L, Li N, Xu C, Zhong S, Lin X, Yang J, Zhou T, Yuliang A, Wu Y, Chen YR, Cao X, Zemach A, Rustgi S, von Wettstein D, Liu B (2014) Mutation of a major CG methylase in rice causes genomewide hypomethylation, dysregulated genome expression, and seedling lethality. Proc Natl Acad Sci USA 111(29):10642-10647. https://doi.org/10.1073/pnas.1410761111

Jung H, Chung PJ, Park S-H, Redillas MCFR, Kim YS, Suh J-W, Kim J-K (2017) Overexpression of OsERF48 causes regulation of OsCML16, a calmodulin-like protein gene that enhances root growth and drought tolerance. Plant Biotechnol J 15(10):12951308. https://doi.org/10.1111/pbi.12716

Kankel MW, Ramsey DE, Stokes TL, Flowers SK, Haag JR, Jeddeloh JA, Riddle NC, Verbsky ML, Richards EJ (2003) Arabidopsis MET1 cytosine methyltransferase mutants. Genetics 163(3):1109-1122

Kim Y, Chung YS, Lee E, Tripathi P, Heo S, Kim K-H (2020a) Root response to drought stress in rice (Oryza sativa $\mathrm{L}$.). Int J Mol Sci 21(4):1513. https://doi.org/10.3390/ijms21041513

Kim YK, Chae S, Oh NI, Nguyen NH, Cheong JJ (2020b) Recurrent drought conditions enhance the induction of drought stress memory genes in Glycine max L. Front Genet 9(11):576086. https:// doi.org/10.3389/fgene.2020.576086

Krzywinski M, Schein J, Birol I, Connors J, Gascoyne R, Horsman D, Jones SJ, Marra MA (2009) Circos: an information aesthetic for comparative genomics. Genome Res 19(9):1639-1645. https:// doi.org/10.1101/gr.092759.109

Law JA, Jacobsen SE (2010) Establishing, maintaining and modifying DNA methylation patterns in plants and animals. Nat Rev Genet 11(3):204-220. https://doi.org/10.1038/nrg2719

Lee D-K, Jung H, Jang G, Jeong JS, Kim YS, Ha S-H, Do Choi Y, Kim J-K (2016) Overexpression of the OsERF71 transcription factor alters rice root structure and drought resistance. Plant Physiol 172(1):575-588. https://doi.org/10.1104/pp.16.00379

Li P, Yang H, Wang L, Liu H, Huo H, Zhang C, Liu A, Zhu A, Hu J, Lin Y, Liu L (2019) Physiological and transcriptome analyses reveal short-term responses and formation of memory under drought stress in rice. Front Genet 10:55. https://doi.org/10.3389/ fgene.2019.00055

Li R, Hu F, Li B, Zhang Y, Chen M, Fan T, Wang T (2020) Whole genome bisulfite sequencing methylome analysis of mulberry (Morus alba) reveals epigenome modifications in response to drought stress. Sci Rep 10(1):8013. https://doi.org/10.1038/ s41598-020-64975-5

Lindroth AM (2001) Requirement of CHROMOMETHYLASE3 for maintenance of CpXpG methylation. Science 292(5524):20772080. https://doi.org/10.1126/science.1059745

Liu N, Staswick PE, Avramova Z (2016) Memory responses of jasmonic acid-associated Arabidopsis genes to a repeated dehydration stress: transcriptional memory of JA genes to dehydration. Plant Cell Environ 39(11):2515-2529. https://doi.org/10.1111/ pce. 12806

Miura A, Yonebayashi S, Watanabe K, Toyama T, Shimada H, Kakutani T (2001) Mobilization of transposons by a mutation abolishing full DNA methylation in Arabidopsis. Nature 411(6834):212214. https://doi.org/10.1038/35075612

Nadarajah K, Kumar IS (2019) Drought response in rice: the miRNA story. Int J Mol Sci 20(15):3766. https://doi.org/10.3390/ijms2 0153766

Nakashima K, Tran L-SP, Van Nguyen D, Fujita M, Maruyama K, Todaka D, Ito Y, Hayashi N, Shinozaki K, Yamaguchi-Shinozaki K (2007) Functional analysis of a NAC-type transcription factor 
OsNAC6 involved in abiotic and biotic stress-responsive gene expression in rice: rice OsNAC6 functions in stress responses. Plant J 51(4):617-630. https://doi.org/10.1111/j.1365-313X.2007. 03168.x

Niederhuth CE, Bewick AJ, Ji L, Alabady MS, Kim KD, Li Q, Rohr NA, Rambani A, Burke JM, Udall JA, Egesi C, Schmutz J, Grimwood J, Jackson SA, Springer NM, Schmitz RJ (2016) Widespread natural variation of DNA methylation within angiosperms. Genom Biol 17(1):194. https://doi.org/10.1186/s13059-016-1059-0

Rajkumar MS, Shankar R, Garg R, Jain M (2020) Bisulphite sequencing reveals dynamic DNA methylation under desiccation and salinity stresses in rice cultivars. Genomics 112(5):3537-3548. https://doi.org/10.1016/j.ygeno.2020.04.005

Redillas MCFR, Jeong JS, Kim YS, Jung H, Bang SW, Choi YD, Ha S-H, Reuzeau C, Kim J-K (2012) The overexpression of OsNAC9 alters the root architecture of rice plants enhancing drought resistance and grain yield under field conditions: OsNAC9 improves drought resistance and grain yield in rice. Plant Biotechnol J 10(7):792-805. https://doi.org/10.1111/j.1467-7652.2012.00697.x

Riemann M, Dhakarey R, Hazman M, Miro B, Kohli A, Nick P (2015) Exploring jasmonates in the hormonal network of drought and salinity responses. Front Plant Sci. https://doi.org/10.3389/fpls. 2015.01077

Sahebi M, Hanafi MM, Rafii MY, Mahmud TMM, Azizi P, Osman M, Abiri R, Taheri S, Kalhori N, Shabanimofrad M, Miah G, Atabaki $\mathrm{N}$ (2018) Improvement of drought tolerance in rice (Oryza sativa L.): genetics, genomic tools, and the WRKY gene family. BioMed Res Int 2018:1-20. https://doi.org/10.1155/2018/3158474

Santosh Kumar VV, Yadav SK, Verma RK, Shrivastava S, Ghimire O, Pushkar S, Rao MV, Senthil Kumar T, Chinnusamy V (2020) The abscisic acid receptor OsPYL6 confers drought tolerance to indica rice through dehydration avoidance and tolerance mechanisms. J Exp Bot. https://doi.org/10.1093/jxb/eraa509

Shen H, Liu C, Zhang Y, Meng X, Zhou X, Chu C, Wang X (2012) OsWRKY30 is activated by MAP kinases to confer drought tolerance in rice. Plant Mol Biol 80(3):241-253. https://doi.org/10. 1007/s11103-012-9941-y

Tabassum T, Farooq M, Ahmad R, Zohaib A, Wahid A, Shahid M (2018) Terminal drought and seed priming improves drought tolerance in wheat. Physiol Mol Biol Plants 24(5):845-856. https:// doi.org/10.1007/s12298-018-0547-y

Todaka D, Shinozaki K, Yamaguchi-Shinozaki K (2015) Recent advances in the dissection of drought-stress regulatory networks and strategies for development of drought-tolerant transgenic rice plants. Front Plant Sci. https://doi.org/10.3389/fpls.2015.00084
Virlouvet L, Avenson TJ, Du Q, Zhang C, Liu N, Fromm M, Avramova Z, Russo SE (2018) Dehydration stress memory: gene networks linked to physiological responses during repeated stresses of Zea mays. Front Plant Sci 9:1058. https://doi.org/10.3389/fpls.2018. 01058

Wang W-S, Pan Y-J, Zhao X-Q, Dwivedi D, Zhu L-H, Ali J, Fu B-Y, Li Z-K (2011) Drought-induced site-specific DNA methylation and its association with drought tolerance in rice (Oryza sativa L.). J Exp Bot 62(6):1951-1960. https://doi.org/10.1093/jxb/erq391

Wang W, Qin Q, Sun F, Wang Y, Xu D, Li Z, Fu B (2016) Genomewide differences in DNA methylation changes in two contrasting rice genotypes in response to drought conditions. Front Plant Sci. https://doi.org/10.3389/fpls.2016.01675

Xia H, Huang W, Xiong J, Yan S, Tao T, Li J, Wu J, Luo L (2017) Differentially methylated epiloci generated from numerous genotypes of contrasting tolerances are associated with osmotic-tolerance in rice seedlings. Front Plant Sci. https://doi.org/10.3389/fpls.2017. 00011

Zhang C, Peng X, Guo X, Tang G, Sun F, Liu S, Xi Y (2018a) Transcriptional and physiological data reveal the dehydration memory behavior in switchgrass (Panicum virgatum L.). Biotechnol Biofuels 11(1):91. https://doi.org/10.1186/s13068-018-1088-x

Zhang J, Zhang H, Srivastava AK, Pan Y, Bai J, Fang J, Shi H, Zhu J-K (2018b) Knockdown of rice microRNA166 confers drought resistance by causing leaf rolling and altering stem xylem development. Plant Physiol 176(3):2082-2094. https://doi.org/10.1104/ pp.17.01432

Zheng X, Chen B, Lu G, Han B (2009) Overexpression of a NAC transcription factor enhances rice drought and salt tolerance. Biochem Biophys Res Commun 379(4):985-989. https://doi.org/10.1016/j. bbrc.2008.12.163

Zheng X, Chen L, Li M, Lou Q, Xia H, Wang P, Li T, Liu H, Luo L (2013) Transgenerational variations in DNA methylation induced by drought stress in two rice varieties with distinguished difference to drought resistance. PLoS ONE 8(11):e80253. https://doi. org/10.1371/journal.pone.0080253

Publisher's Note Springer Nature remains neutral with regard to jurisdictional claims in published maps and institutional affiliations. 